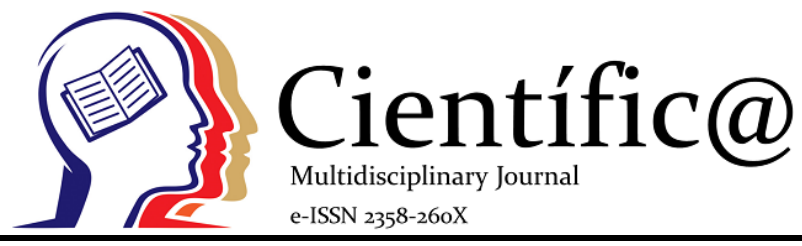

\title{
Sensores Termohigrométricos: Procedimentos para montagem e calibração
}

Thermo-Hygrometric Sensors: Assembly and Calibration Procedures

\author{
Marcos de Oliveira Valin Jr¹; Flávia Maria de Moura Santos²; João Basso Marques ${ }^{3}$; Karyn
} Ferreira Antunes Ribeiro 4

\footnotetext{
${ }^{1}$ IFMT e-mail: marcos.valin@cba.ifmt.edu.br - Lattes: http://lattes.cnpq.br/1988817143994600

${ }^{2}$ UFMT e-mail: flavia_mms@hotmail.com Lattes: http://lattes.cnpq.br/4000857814123856

${ }^{3}$ UFMT e-mail: jbassofisico@gmail.com Lattes: http://lattes.cnpq.br/7504881282942727

4 IFMT e-mail: karyn.ribeiro@cba.ifmt.edu.br Lattes: http://lattes.cnpq.br/1028794796688155
}

\section{Info}

Recebido: $28 / 05 / 2019$

Publicado: $31 / 05 / 2019$

DOI: 10.29247/2358-260X.2019v6i1.p134-143

ISSN: 2358-260X

\section{Palavras-Chave}

Datalloger, temperatura do ar, umidade relativa do ar, sensores.

\section{Keywords:}

DataLogger, air temperature, relative humidity, sensors.

\section{Abstract}

This article presents procedures for assembly and calibration of five thermohygrometric sensors, which were developed for measurement and studies in urban environments, specifically in mobile transect used in doctoral thesis research. The sensors used in this research were the DHT22 model (measure the air temperature and the relative humidity of the air). The datalogger was programmed on a Raspberry Pi 3 microcontroller. Observing the need for calibration, the sensors were registered in the same settings and environmental conditions as the standard sensor (HOBO U12-012). Statistical analyzes (multiple comparisons) were performed, which included the real need for the

calibration of the five sensors. It was concluded that the thermo-hygrometer made in this work, after being calibrated, presented reliable and good quality measurements. And that the methodology proved to be efficient and reliable for the development and calibration of the sensors used, contributing to other studies related to the measurements of microclimatological variables being used and used.

\section{Resumo}

Este artigo apresenta procedimentos para montagem e calibração de cinco sensores termohigrométricos, que foram desenvolvidos para a medição e estudos em ambientes urbanos, especificamente em transecto móvel utilizados em pesquisa de tese de doutorado. Os sensores utilizados nessa pesquisa foram do modelo DHT22 (medem a temperatura do ar e a umidade relativa do ar). 0 datalogger (registrador de dados) foi programado em placa microcontroladora Raspberry Pi 3. Observando-se a necessidade de aferição, os sensores foram registrados nas mesmas configurações e condições ambientais que o sensor padrão (HOBO U12-012). Foram realizadas análises estatísticas (comparações múltiplas) que constaram a real necessidade da calibração dos cinco sensores. Concluise que o termo-higrômetro confeccionado neste trabalho, após ser calibrado, apresentou medidas confiáveis e de boa qualidade. E que a metodologia se mostrou eficaz e confiável para o desenvolvimento e calibração dos sensores utilizados, contribuindo para que em outros estudos relacionados às medidas de variáveis da microclimatológicas sejam empregados e utilizados. 


\section{INTRODUÇÃO}

Estudos relacionados a climatologia são imprescindíveis para garantir a qualidade de vida das pessoas e são realizados são realizados desde a época da revolução industrial onde ocorriam surtos de doenças por falta de infraestrutura, e nos dias mais recentes proporcionando moradias de qualidade e conforto aos trabalhadores. São exemplos desses estudos as aplicações na avaliação de ilhas de calor, projetos de edificações, conforto ambiental, planejamento urbano e até para controle epidemiológico de vetores causadores de doenças como a dengue.

Nessas pesquisas são indispensáveis a realização de trabalhos em campo, visando à obtenção de dados para serem analisados, envolvendo mais do que somente observação e análise, normalmente visam à obtenção de dados de temperatura do ar, e umidade relativa do ar, precipitação, radiação, entre outros.

Esses dados só podem ser obtidos através do uso de equipamentos e sensores específicos que para garantir a qualidade e padronização dos dados devem seguir procedimentos de instalação e estarem calibrados.

De acordo com Neves et al (2015) geralmente os sensores são comercializados com certificações apenas se forem utilizados com o logger apropriado, do mesmo fabricante, aumentando significativamente o orçamento das pesquisas.

Dados de temperatura e de umidade relativa do ar são utilizados como indicativos das características gerais da área de estudo, como por exemplo, sobre o período sazonal, a localização latitudinal, a proximidade com o mar, ou mesmo os tipos de uso e ocupação do solo ali predominantes (Serafini Júnior et al., 2014).

$\mathrm{O}$ monitoramento da temperatura $\mathrm{e}$ umidade do ar são importantes para o estudo do clima urbano, pois conforme Maciel (2011) a substituição da cobertura natural do solo por materiais como o asfalto e o concreto modificam o balanço de energia nas áreas urbanas.

Um dos desafios atuais na área de pesquisa de clima urbano refere-se à padronização dos procedimentos de coleta e análise de dados (OKE, 2005).

Como forma de fornecer subsídio aos estudos que utilizam de sensores termohigrométricos, em especial os de clima urbano, é proposto como objetivo geral apresentar a metodologia de desenvolvimento e processo de análise estatística para calibrar sensores termohigrômetros para uso em pesquisa climatológica.

\section{MATERIAIS E MÉTODOS}

2.1 Sensores e datalloger utilizados

Para Barbosa, et al. (2008) a obtenção de dados através do procedimento por medição foi facilitada pelo surgimento de equipamentos registradores e armazenadores eletrônicos de temperatura e outros sinais (data-loggers).

Os sensores utilizados foram o do modelo DHT22 (Figura 1 - item a) para medir a temperatura nas escalas de -40 a $125^{\circ} \mathrm{C}$ e a umidade do ar nas faixas de 0 a $100 \%$. O datalogger (Registrador de dados) foi programado em placa microcontroladora Raspberry Pi 3 (Figura 1 -item 
b), cujas especificações estão apresentadas na

Tabela 1.

Tabela 1 - Especificações

\begin{tabular}{|c|c|}
\hline Sensor DHT22 & Processador Raspberry Pi 3 Modelo B \\
\hline - Tensão de alimentação: 3 - 5,5VDC; & - Tensão de operação: Micro USB 5V/2A; \\
\hline - Faixa de Corrente: $0,5 \sim 2,5 \mathrm{~mA}$; & - SoC: Broadcom BCM2837 64bits; \\
\hline - Sinal de saída digital (single-bus); & - Arquitetura: Quad-core ARMv8 Cortex-A53; \\
\hline - Período de detecção: 2s; & - Velocidade: 1.2GHz; \\
\hline - Faixa de operação de umidade: $0 \sim 100 \%$; & - Memória: 1Gb \\
\hline - Precisão de Umidade: $2 \% \mathrm{RH}$; & - GPU: Broadcom VideoCore IV 3M (400 \\
\hline - Faixa de operação da temperatura: -40 a $125^{\circ}$ & $\mathrm{MHz}$; \\
\hline Celsius; & - Adaptador Wifi 802.11n Wireless LAN: \\
\hline - Precisão de Temperatura: $0,20^{\circ} \mathrm{C}$; & integrado; \\
\hline \multirow[t]{2}{*}{ - Dimensões totais (CxLxA): 36,5x15,2x9,4mm; } & - Bluetooth 4.1 BLE: integrado; \\
\hline & - Dimensões (CxLxA): 85x56x17mm; \\
\hline
\end{tabular}

A proposta de utilização do equipamento após a calibração consiste em transecto e para isso é imprescindível a utilização do Módulo GPS + Antena (Figura 1 - item c), para o registro de cada coordenada relacionada com a temperatura e umidade registrada. O equipamento utilizado é do modelo: GY-GPS6MV2 Ublox, Tensão de

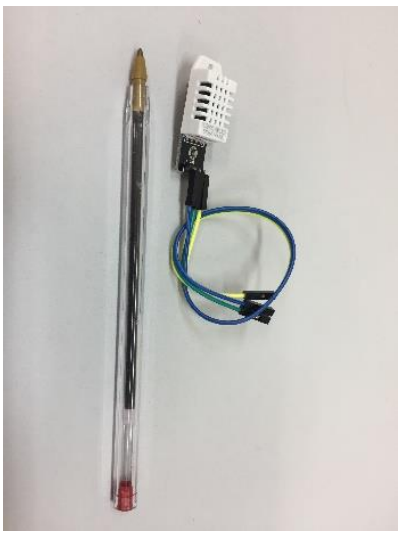

(a)

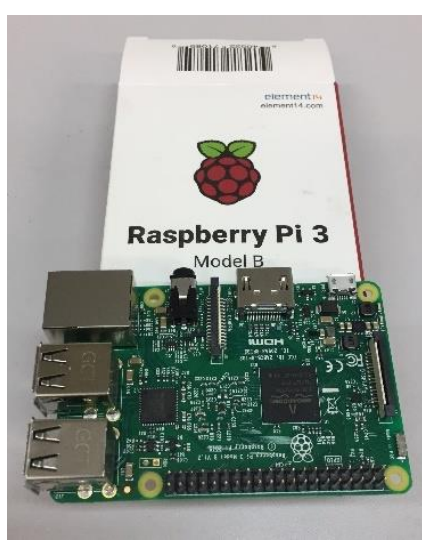

(b) trabalho: 3,3 a 5V DC, Interface serial de 3,3V, Taxa de transmissão padrão: 9600, Dimensões do módulo (CxLxA): 30x23x4mm e Dimensões da antena (CxLxA): 25x25x8mm.

Figura 1 - Componentes utilizados: (a) Sensor DHT22 6; (b) Processador Raspberry Pi 3 6; (c) GPS 6 
Para elaboração do Datalogger também foram utilizados os seguintes componentes:

a) Conversor Analógico Digital 12 Bits ADC: Modelo ADS1015; Tensão de operação: 2V 5.5V, Consumo: 150uA; N. de canais: 4 simples ou 2 diferenciais; Comunicação: I2C; Taxa de amostragem: programável de 8sps à 860sps; Dimensões (CxLxE): 25,7x9x2.5mm; Peso: $1 \mathrm{~g}$.

O Conversor Analógico
Digital ADS1015,
internacionalmente conhecido
por ADC, é um pequeno
dispositivo digital capaz de
realizar a conversão de sinais
analógicos em sinais digitais
quando aplicado em projetos
com microcontroladores sem
conversor integrado ou
quando busca-se maior
precisão ADC. (Usina Ind.
Comércio e Importação,
2018)

b) Módulo Display OLED": Interface: SPI; Tensão: 3,3 a 5V; Resolução: 128x64 pixels; Tela: 0,96"; Dimensões (CxLxA): 28x28x12mm; Dimensões da tela (CxL): 27x15mm; Peso: 3,4g.

c) Módulo Relógio Tempo Real RTC Compacto - DS1307: Tensão de trabalho externa: 5V; Armazenamento de memória: 24C32 EEPROM I2C 32K; Memória RAM não volátil: 56 byts; Protocolo de acesso I2C; Dimensões (CxLxA): 28x27x6,1mm; Peso: 3g.

Com o crescente desenvolvimento de projetos de automação, por exemplo, através das placas microcontroladoras: Arduino, AVR, PIC, ARM, são necessários acessórios para dar as placas diferentes funcionalidades e aperfeiçoamentos. O Módulo Relógio Tempo Real (RTC) DS1307 é um módulo compacto que possui a capacidade de calcular precisamente o tempo, realizando a contagem exata dos segundos, minutos, horas, bem como, dos dias, semanas, meses e anos. (Usina Ind. Comércio e Importação, 2018)

d) Bateria CR2032 3V de Lithium / Pilha CR2032 - GoldenPower;

e) Cartão de Memória: Marca: SanDisk; Modelo: $\quad$ SDSQUNB-016G-GN3MN; Tipo: MicroSDHC; Capacidade: 16GB; Classe: 10; Velocidade: taxa de leitura até $48 \mathrm{MB} / \mathrm{s}$.

f) Cabo chato 4 vias;

g) Conectores e keystone RJ11.

Os programas foram escritos na linguagem Python. Os scripts são iniciados automaticamente na inicialização do Raspberry Pi, através de um agendamento de tarefas do sistema operacional Linux. Para a visualização e monitoramento instantâneo foi integrado um Display para facilitar o processo de operação (Erro! Fonte de referência não encontrada. -item a). Considerando as especificidades da utilização em transecto móvel, os sensores são conectados ao datalogger por meio de conectores RJ11 (Erro! Fonte de referência não encontrada. - item b). 

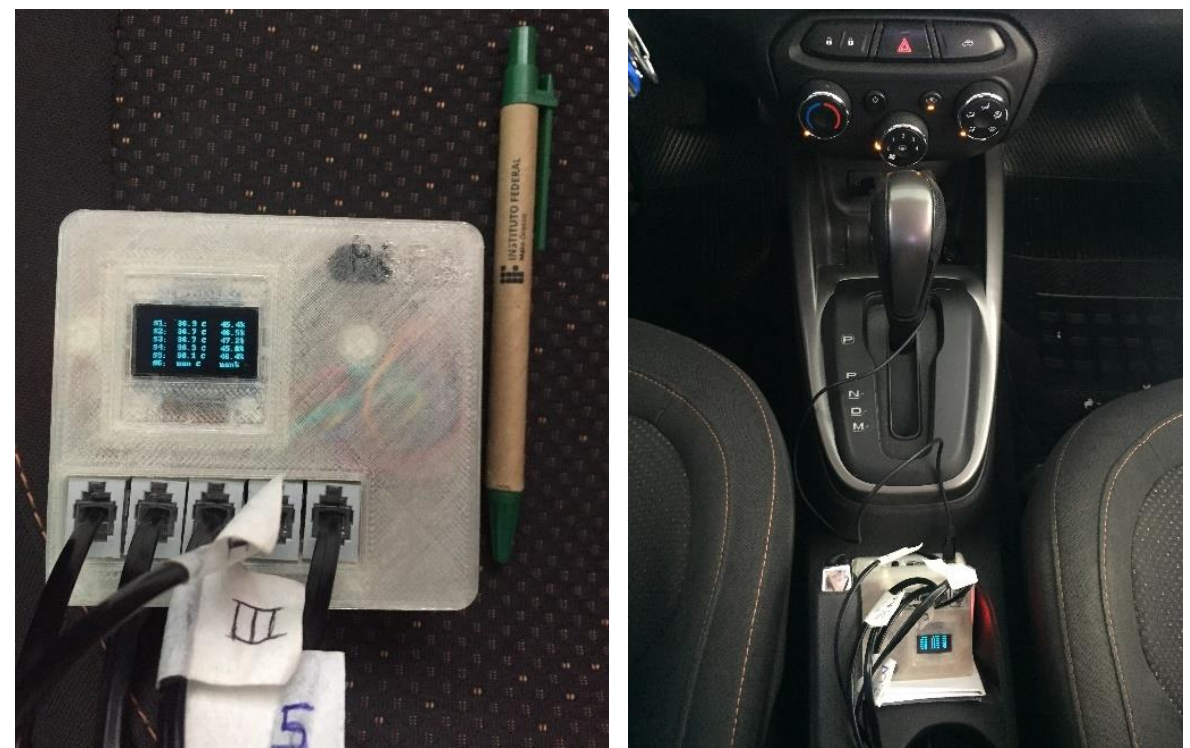

Figura 2 - Datalloger: (a) detalhe do visor; (b) Posicionamento no veículo com alimentação na porta USB

\subsection{Calibração e procedimentos estatísticos}

Considerando a necessidade de calibração dos sensores, os mesmos foram ligados nas mesmas configurações e condições ambientais controladas com um sensor padrão calibrado, o do modelo HOBO U12-012, de fabricação da Onset Compter Corporation.

A primeira análise dos dados foi a verificação dos intervalos de confiança, para verificar a real necessidade da calibração, com análise dos dados da média, erro padrão, limites inferior e superior dos intervalos de confiança.

O valor do erro padrão apresentado é puramente o desvio padrão (métrica de dispersão dos dados em torno da média) dividido pela raiz quadrada do $\mathrm{N}$ amostral, ou seja, é uma relação: dispersão de dados / tamanho da amostra. Em estatística há o pressuposto de que existe uma média real na população, e as amostras coletadas tentam inferir essa média.

$\mathrm{Na}$ sequência para verificar a significância das diferenças entre os sensores, foi então aplicado o teste não paramétrico de Kruskall Wallis, sendo comprovado que as diferenças eram significativas, nos casos em que $\mathrm{p}<0,001$. Porém esse teste só mostra que há diferenças, mas não aponta onde está a diferença.

Para ver exatamente quais sensores diferem e quais são iguais (só se faz quando o primeiro teste for significativo para apontar onde estão as diferenças encontradas pelo primeiro teste) foi realizado o teste de Comparações Múltiplas.

Com base nos testes realizados, realiza-se a avaliação dos valores médios dos sensores, assim temos na diferença do sensor padrão para os demais a calibração de cada um dos sensores no que concerne ao dado medido.

Assim, somasse ou subtraísse dos valores de cada sensor em relação ao sensor padrão os valores para dizer que foi calibrado.

As análises foram realizadas em separado para os dados da Temperatura do Ar e da Umidade Relativa do Ar. 
3 CALIBRAÇÃO DOS SENSORES

3.1 Sensores de temperatura
O gráfico da Figura 3 apresenta os valores obtidos na leitura inicial de Temperatura do ar (antes da calibração), demonstrando que todos os sensores divergem do sensor padrão.

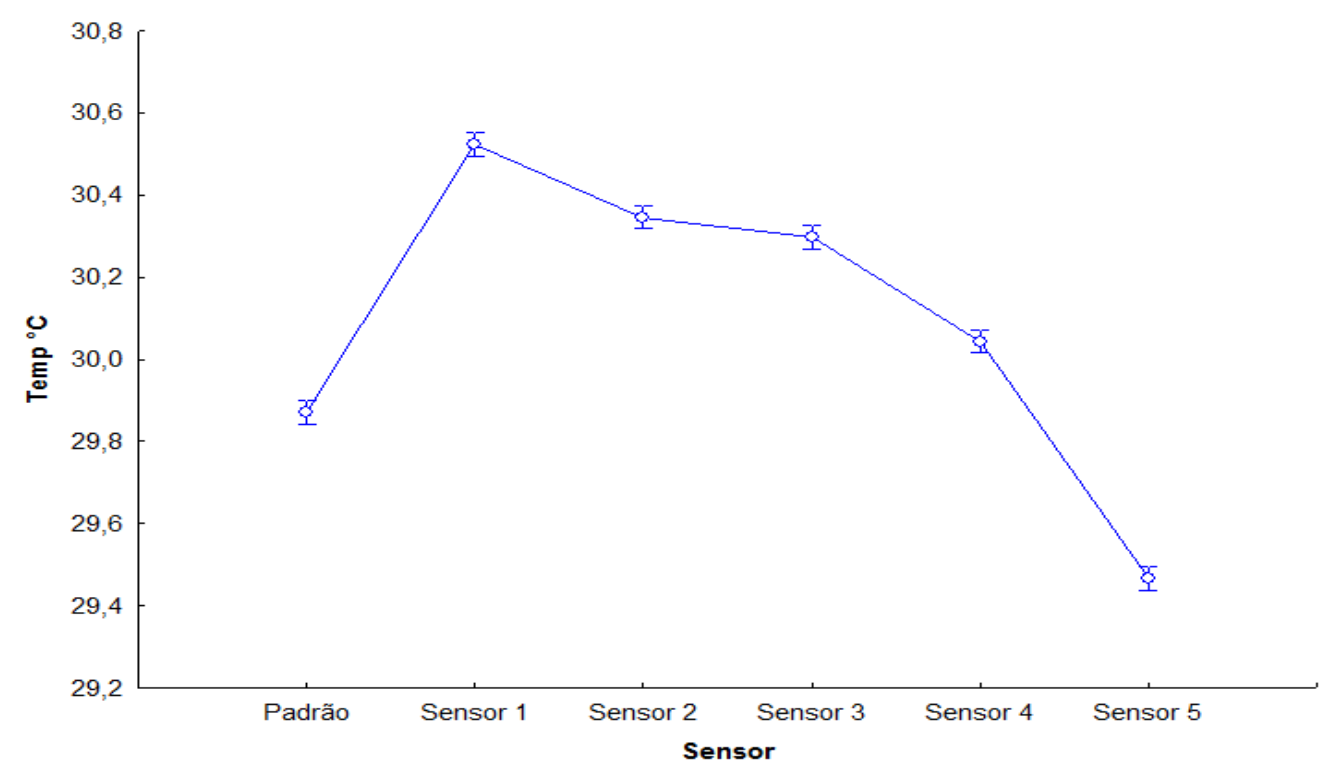

Figura 3 - Intervalo de confiança dos sensores para Temperatura

Em números o gráfico fica como média, erro padrão, limites inferior e superior dos apresentado na Erro! Fonte de referência não intervalos de confiança e quantidade de dados para encontrada.. As colunas apresentam da esquerda cada estimativa. para direita: identificação do sensor, temperatura

Tabela 2 - Intervalo de confiança dos sensores para temperatura

\begin{tabular}{|c|c|c|c|c|c|}
\hline Sensor & $\begin{array}{l}\text { Temp. }{ }^{\circ} \mathrm{C} \\
\text { Média }\end{array}$ & $\begin{array}{c}\text { Temp. }{ }^{\circ} \mathrm{C} \\
\text { Erro Padrão }\end{array}$ & $\begin{array}{l}\text { Temp. }{ }^{\circ} \mathrm{C} \\
(-95,00 \%)\end{array}$ & $\begin{array}{l}\text { Temp. }{ }^{\circ} \mathrm{C} \\
(+95,00 \%)\end{array}$ & $\mathbf{N}$ \\
\hline Padrão & 29,87 & 0,015 & 29,84 & 29,90 & 1850 \\
\hline Sensor 1 & 30,52 & 0,015 & 30,50 & 30,55 & 1850 \\
\hline Sensor 2 & 30,35 & 0,015 & 30,32 & 30,37 & 1850 \\
\hline Sensor 3 & 30,30 & 0,015 & 30,27 & 30,33 & 1850 \\
\hline Sensor 4 & 30,04 & 0,015 & 30,02 & 30,07 & 1850 \\
\hline Sensor 5 & 29,47 & 0,015 & 29,44 & 29,49 & 1850 \\
\hline
\end{tabular}

No caso, evidencia que a média na amostra

O sensor 1 é o que mais diferiu do sensor padrão e o sensor 4 é o que está mais próximo (apenas 0,17 graus Celsius de diferença). 
0,015 indica que as médias dos sensores são muitíssimo próximas da média "real".

Para verificar a significância das diferenças entre os sensores, foi então aplicado o teste não paramétrico de Kruskall Wallis, apresentado na
Erro! Fonte de referência não encontrada., sendo comprovado que as diferenças eram significativas $(\mathrm{p}<0,001)$, porém esse teste só mostra que há diferenças, mas não aponta onde está a diferença.

Tabela 3 - Teste de Kruskall Wallis para Temperatura

\begin{tabular}{|c|c|c|c|}
\hline \multicolumn{4}{|c|}{ Sensor Kruskal-Wallis test: $H(5, N=11100)=2342,616 p<0,001$} \\
\hline & Code & Valid - N & Sum of - Ranks \\
\hline Padrão & 101 & 1850 & 8378927 \\
\hline Sensor 1 & 102 & 1850 & 13740885 \\
\hline Sensor 2 & 103 & 1850 & 12296820 \\
\hline Sensor 3 & 104 & 1850 & 11841186 \\
\hline Sensor 4 & 105 & 1850 & 9774132 \\
\hline Sensor 5 & 106 & 1850 & 5578601 \\
\hline
\end{tabular}

Para ver exatamente quais sensores diferem e quais são foi realizado o teste de Comparações Múltiplas, que mostra que apenas o sensor 2 e o sensor 3 concordavam entre si (não diferem estatisticamente entre si) todos os demais diferem uns dos outros conforme demonstrado na Tabela 4.

Tabela 4 - Comparações múltiplas após Teste de Kruskall Wallis para Temperatura

\begin{tabular}{|c|c|c|c|c|c|c|}
\hline \multicolumn{7}{|c|}{ Multiple Comparisons p values (2-tailed); $\mathrm{H}(5, \mathrm{~N}=11100)=2342,616 \mathrm{p}=0,000$} \\
\hline & $\begin{array}{l}\text { Padrão - } \\
\text { R:4529,1 }\end{array}$ & $\begin{array}{c}\text { Sensor } 1- \\
\text { R:7427,5 }\end{array}$ & $\begin{array}{c}\text { Sensor } 2- \\
\text { R:6646,9 }\end{array}$ & $\begin{array}{c}\text { Sensor } 3- \\
\text { R:6400,6 }\end{array}$ & $\begin{array}{c}\text { Sensor } 4- \\
\text { R:5283,3 }\end{array}$ & $\begin{array}{l}\text { Sensor } 5 \\
\text { R:3015,5 }\end{array}$ \\
\hline Padrão & - & $<0,0001$ & $<0,0001$ & $<0,0001$ & $<0,0001$ & $<0,0001$ \\
\hline Sensor 1 & $<0,0001$ & - & $<0,0001$ & $<0,0001$ & $<0,0001$ & $<0,0001$ \\
\hline Sensor 2 & $<0,0001$ & $<0,0001$ & - & 0,2911 & $<0,0001$ & $<0,0001$ \\
\hline Sensor 3 & $<0,0001$ & $<0,0001$ & 0,2911 & - & $<0,0001$ & $<0,0001$ \\
\hline Sensor 4 & $<0,0001$ & $<0,0001$ & $<0,0001$ & $<0,0001$ & - & $<0,0001$ \\
\hline Sensor 5 & $<0,0001$ & $<0,0001$ & $<0,0001$ & $<0,0001$ & $<0,0001$ & - \\
\hline
\end{tabular}

Com base nos testes realizados, ficou valores médios dos sensores, assim temos na comprovada a necessidade da calibração para os diferença do sensor padrão para os demais a dados da temperatura, constando na Erro! Fonte calibração de cada um no que concerne à de referência não encontrada. a avaliação dos temperatura. 
Tabela 5 - Calibração dos valores de Temperatura

\begin{tabular}{ccc}
\hline Sensor & Temp $\left({ }^{\circ} \mathbf{C}\right)$ Média & Diferença do Sensor Padrão \\
\hline Padrão & 29,87 & 0 \\
\hline Sensor 1 & 30,52 & $-0,65$ \\
\hline Sensor 2 & 30,35 & $-0,48$ \\
\hline Sensor 3 & 30,30 & $-0,43$ \\
\hline Sensor 4 & 30,04 & $-0,17$ \\
\hline Sensor 5 & 29,47 & $+0,40$ \\
\hline
\end{tabular}

Assim, o Sensor 1 deve ser calibrado com a subtração de 0,65 aos valores, o Sensor 2 de 0,48, o Sensor 3 de 0,43, o Sensor 4 de 0,17 e para Sensor 5 deve-se somar 0,4 nas medidas.
3.2 Sensores de umidade relativa do ar

O gráfico da Figura 4 apresenta os valores obtidos na leitura inicial da Umidade Relativa do $\operatorname{Ar}$ (antes da calibração), demonstrando que todos os sensores divergem do sensor padrão.

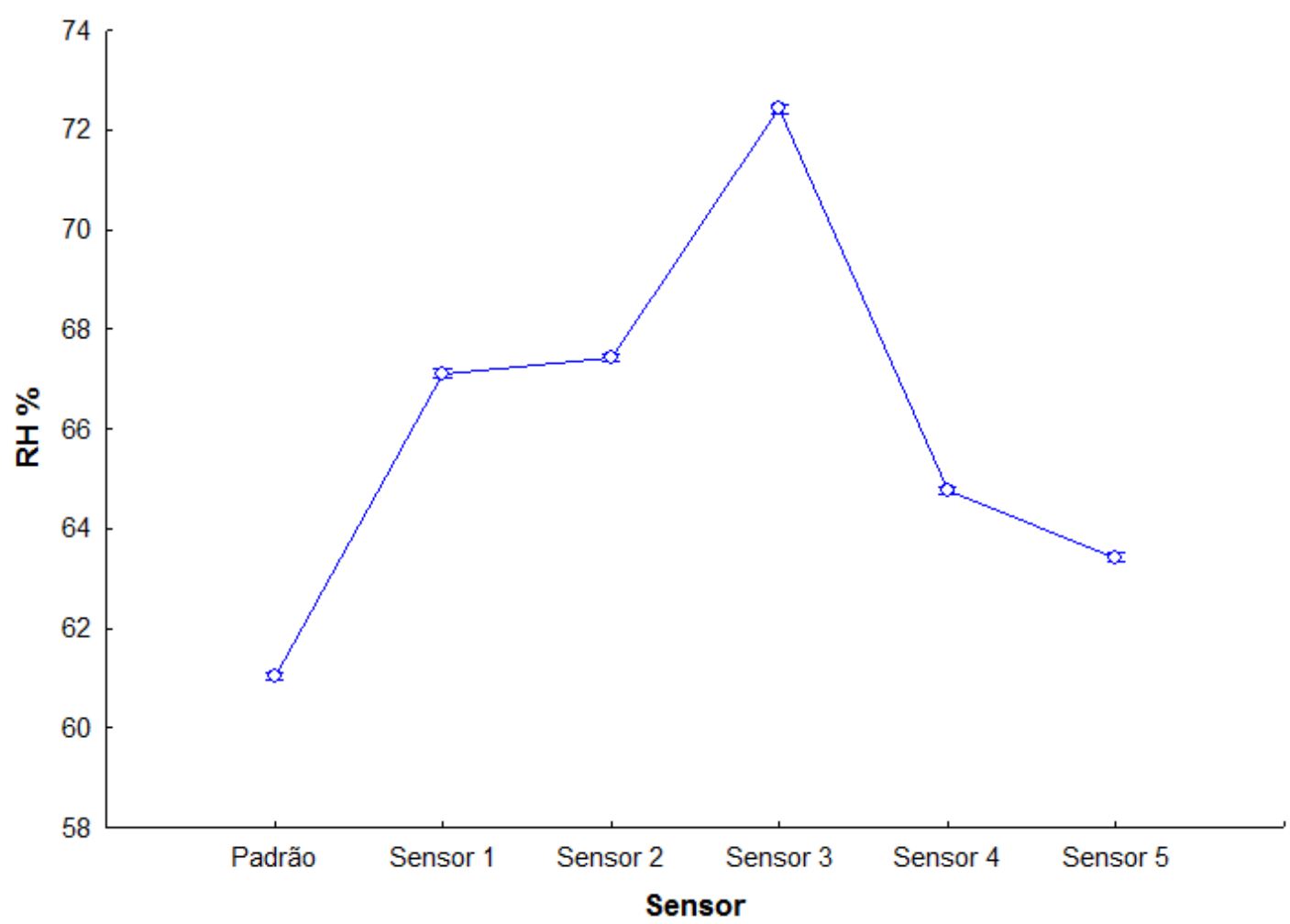

Figura 4 - Intervalo de confiança dos sensores para Umidade Relativa

Em números, o gráfico fica como superior dos intervalos de confiança e quantidade apresentado na Tabela 6 . As colunas apresentam da de dados para cada estimativa. esquerda para direita: identificação do sensor, temperatura média, erro padrão, limites inferior e 
Tabela 6 - Intervalo de confiança dos sensores para umidade relativa

\begin{tabular}{lcccccc}
\hline Sensor & $\begin{array}{c}\text { HR \% - } \\
\text { Média }\end{array}$ & $\begin{array}{c}\text { HR \% } \\
\text { Erro Padrão }\end{array}$ & $\begin{array}{c}\text { HR \% } \\
\mathbf{( - 9 5 , 0 0 \% )}\end{array}$ & $\begin{array}{c}\text { HR \% } \\
\mathbf{( + 9 5 , 0 0 \% )}\end{array}$ & N \\
\hline $\mathbf{1}$ & Padrão & 61,03 & 0,043 & 60,94 & 61,11 & 1850 \\
\hline $\mathbf{2}$ & Sensor 1 & 67,11 & 0,043 & 67,03 & 67,20 & 1850 \\
\hline $\mathbf{3}$ & Sensor 2 & 67,43 & 0,043 & 67,35 & 67,51 & 1850 \\
\hline $\mathbf{4}$ & Sensor 3 & 72,42 & 0,043 & 72,34 & 72,50 & 1850 \\
\hline $\mathbf{5}$ & Sensor 4 & 64,76 & 0,043 & 64,68 & 64,85 & 1850 \\
\hline $\mathbf{6}$ & Sensor 5 & 63,42 & 0,043 & 63,33 & 63,50 & 1850 \\
\hline
\end{tabular}

Para umidade relativa, o Sensor 3 é o que mais difere do sensor padrão, sendo o Sensor 5 o mais próximo.
O teste de Kruskall Wallis, Tabela 7, mostra que as diferenças entre os valores de umidade são significativas $(\mathrm{p}<0,001)$.

Tabela 7 - Teste de Kruskall Wallis para Umidade Relativa

\begin{tabular}{|c|c|c|c|}
\hline \multicolumn{4}{|c|}{ Sensor Kruskal-Wallis test: $\mathrm{H}(5, \mathrm{~N}=11100)=8714,587 \mathrm{p}<0,001$} \\
\hline & Code & Valid - N & Sum of - Ranks \\
\hline Padrão & 101 & 1850 & 2631502 \\
\hline Sensor 1 & 102 & 1850 & 12585925 \\
\hline Sensor 2 & 103 & 1850 & 13154833 \\
\hline Sensor 3 & 104 & 1850 & 18739134 \\
\hline Sensor 4 & 105 & 1850 & 8578467 \\
\hline Sensor 5 & 106 & 1850 & 5920691 \\
\hline
\end{tabular}

O teste a posteriori de Comparações 2 concordam entre si, todos os demais diferem uns Múltiplas mostra que apenas o sensor 1 e o sensor dos outros conforme Tabela 8.

Tabela 8 - Comparações múltiplas após Teste de Kruskall Wallis para Umidade Relativa

\begin{tabular}{|c|c|c|c|c|c|c|}
\hline \multicolumn{7}{|c|}{ Multiple Comparisons $\mathrm{p}$ values (2-tailed); $\mathrm{H}(5, \mathrm{~N}=11100)=8714,587 \mathrm{p}<0,001$} \\
\hline & Padrão & Sensor 1 & Sensor 2 & Sensor 3 & Sensor 4 & Sensor 5 \\
\hline & R:1422,4 & R:6803,2 & R:7110,7 & R:10129,0 & R:4637,0 & R:3200,4 \\
\hline Padrão & - & $<0,0001$ & $<0,0001$ & $<0,0001$ & $<0,0001$ & $<0,0001$ \\
\hline Sensor 1 & $<0,0001$ & - & 0,05272 & $<0,0001$ & $<0,0001$ & $<0,0001$ \\
\hline Sensor 2 & $<0,0001$ & 0,0527 & - & $<0,0001$ & $<0,0001$ & $<0,0001$ \\
\hline Sensor 3 & $<0,0001$ & $<0,0001$ & $<0,0001$ & - & $<0,0001$ & $<0,0001$ \\
\hline Sensor 4 & $<0,0001$ & $<0,0001$ & $<0,0001$ & $<0,0001$ & - & $<0,0001$ \\
\hline Sensor 5 & $<0,0001$ & $<0,0001$ & $<0,0001$ & $<0,0001$ & $<0,0001$ & - \\
\hline
\end{tabular}

Com base nos testes realizados ficou temos na diferença do sensor padrão para os comprovada a necessidade da calibração para os demais a calibração de cada um no que concerne à dados da umidade relativa, sendo na Tabela 9 a Umidade Relativa. avaliação dos valores médios dos sensores, assim 
Tabela 9 - Calibração dos valores de Umidade Relativa

\begin{tabular}{ccc}
\hline Sensor & HR \% - Média & Diferença para o Sensor Padrão \\
\hline Padrão & 61,03 & 0 \\
\hline Sensor 1 & 67,11 & $-6,08$ \\
\hline Sensor 2 & 67,43 & $-6,40$ \\
\hline Sensor 3 & 72,42 & $-11,39$ \\
\hline Sensor 4 & 64,76 & $-3,73$ \\
\hline Sensor 5 & 63,42 & $-2,39$ \\
\hline
\end{tabular}

Portanto, o Sensor 1 deve ser calibrado com a subtração de 6,08 aos valores, o Sensor 2 de 6,40, o Sensor 3 de 11,39, o Sensor 4 de 3,73 e para sensor 5 de 2,39 nas medidas.

\section{CONSIDERAÇÕES FINAIS}

O termo-higrômetro construído neste trabalho, após ser submetido ao processo de calibração, apresentou medidas confiáveis de temperatura e umidade relativa do ar.

O procedimento realizado foi executado antes da utilização dos sensores para o objetivo ao qual foi produzido, tese de doutorado, sendo que todas as análises foram realizadas considerando os sensores já calibrados após os procedimentos detalhados.

A metodologia aplicada neste estudo mostrou-se eficaz e confiável para o desenvolvimento e calibração dos sensores utilizados, podendo ser empregada em outros estudos relacionados às medidas de variáveis da Micrometeorologia, Agrometeorologia e Climatologia.

Os resultados obtidos no presente trabalho motivam estudos complementares, com produção de algoritmos abertos para os cálculos dos testes estatísticos com maior facilidade que possam contribuir a pesquisadores.

\section{REFERÊNCIAS}

BARBOSA, M. J.; LAMBERTS, R.; GUTHS, S. Uso de barreiras de radiação para minimizar o erro no registro das temperaturas do ar em edificações. Ambiente Construído. Porto Alegre - RS, v. 8, n. 4, p. 117 - 136, 2008.

MACIEL, C. R. Desenvolvimento e Calibração de um Termohigrômetro para uso em Pesquisas de Micrometeorologia, Agrometeorologia e Climatológica. Dissertação (Mestrado em Física Ambiental), Instituto de Física, Universidade Federal de Mato Grosso, Cuiabá, 2011.

NEVES, G. A. R.; MARQUES, J. B.; NOGUEIRA, J. S.; BUIDES, M. S.; ARRUDA, P. H. Z.; CURADO, L. F.; PALÁCIOS, R.S. Uso de barreiras de radiação para minimizar o erro no registro das temperaturas do ar em edificações. Revista Brasileira de Geografia Física, v. 8, n. 1, p. 136-143, set. 2015.

OKE, T. R. Towards better scientific communication in urban climate. Áustria: Theorical and Applied Climatology, 2005.

SERAFINI JÚNIOR, S.; ALVES, R. R. Miniabrigos meteorológicos: comparação e análise estatística para avaliação de eficiência. GEOUSP - Espaço e Tempo (online), v. 18, p. 198-210, 2014. 
Usina Ind. Comércio e Importação. Usina Info. $2018 . \quad$ Disponível em: https://www.usinainfo.com.br/ . Acesso em:09 de Agosto de 2018. 